
\title{
25 Research Square \\ Protection of flunarizine on ventralis \\ posteromedialis nucleus in rat model of migraine induced by inflammatory soup
}

\section{Wei Ge}

The PLA General Hospital, Medical School of Chinese PLA, Department of Neurology

\section{Lijun Zhao}

Institute of Chemistry, Chinese Academy of Sciences,Beijing National Laboratory for Molecular

Sciences, Key Laboratory of Analytical Chemistry for Living Biosystems

\section{Chao He}

The PLA general Hospital

\section{Qingkui Zhang}

The PLA General Hospital

\section{Yunxia Wang}

The PLA General Hospital

Ruozhuo Liu ( $\nabla$ liuruozhuo301@163.com )

chinese PLA general hospital https://orcid.org/0000-0001-8582-580X

\section{Lanqun Mao}

Institute of Chemistry, The Chinese Academy of Sciences,Beijing National Laboratory for Molecular

Science,Key Laboratoy of Analytical Chemistry for Living Biosystem

\section{Shengyuan Yu}

The PLA General Hospital

\section{Research article}

Keywords: Ventralis posteromedialis nucleus, Migraine, Flunarizine, Green fluorescence protein, CAMPARI

Posted Date: January 1st, 2020

DOI: https://doi.org/10.21203/rs.2.19791/v1

License: (c) (1) This work is licensed under a Creative Commons Attribution 4.0 International License.

Read Full License 


\section{Abstract}

Background: Migraine is a disease closely related to calcium ions and ion channels. Ventralis posteromedialis (VPM) nucleus is an important nucleus in the process of migraine pain signal transmission. Flunarizine has been widely demonstrated as a calcium channel antagonist in the preventive treatment of migraine, but the mechanism of its anti-migraine on VPM is still unclear.

Methods: Part 1. Fifteen transfected male rats were randomly divided into three groups: Flunarizine intervention group, Nimodipine intervention group and Normal saline sham group. Viral expression of fluorescent tracers allows purely anterograde labeling. Following injection of AAV-hSyn-9-CaMPARI-GFP into the physiologically defined area in the VPM nucleus of the thalamus, labeling in the thalamus-cortex had a green appearance. Part 2. Twenty Sprague Dawley male rats were randomly divided into four groups: Single stimulus group, Double stimuli group, Flunarizine intervention group and Nimodipine intervention group. A calcium-selective carbon fiber electrode (CFMEs) is used for online monitoring of electrochemically inactive $\mathrm{Ca}^{2+}$ in the rat brain in vivo. Results: Part 1.GFP Images showed that the VPM nucleus of the thalamus was involved in the signal transmission process of headache. Fluorescence imaging of the VPM nucleus of the thalamus after intervention with flunarizine or nimodipine suggested that it was activated. Part 2. No specific response was observed in the migraine rat model after stimulation with normal saline, extracellular calcium ions increased first and then decreased linearly after each stimulation with inflammatory soup. After the intervention of nimodipine, the curve of calcium changes after IS stimulation was the same as before. While after the intervention of flunarizine, the performance was significantly inconsistent, which was first increased and then maintained a level of concentration or slightly increased. Conclusion: VPM might play an important role in pain signal transmission of migraine and Flunarizine can alleviate such $\mathrm{Ca}^{2+}$ concentration in VPM neurons to exert its anti-migraine effects.

\section{Background}

Migraine is a common, multifactorial, neurovascular diseases that significantly affects individuals and the society[1]. However, the underlying potential pathophysiological mechanisms are poorly understood, the study of headache attack process lacks real - time objective indicators or means. The ventralis posteromedialis (VPM) nucleus is an important part of the head pain relaying nuclear group, which has the function of receiving, integrating and transmitting. Functional neuroimaging and electrophysiological studies have demonstrated its role in the production of head and facial pain[2].

Calcium ions $\left(\mathrm{Ca}^{2+}\right)$ participate in many physiological processes and are indispensable materials. In the central nervous system, calcium ion is a vital intermediate medium in the process of nerve conduction, which engages in the process of neuro-chemical transmission[3]. When the action potential reaches the nerve terminal, voltage dependent $\mathrm{Ca}^{2+}$ channels open and $\mathrm{Ca}^{2+}$ rushes into the neuron terminal due to a greater extracellular concentration ion channel. Familial hemiplegic migraine is an autosomal dominant hereditary calcium ion channel disease, having identified four missense mutation CACNL1A4 gene, the 
gene encoding $P / Q$ voltage-gated calcium channel $₫ 1 A$ subunit[4]. This therefore suggests an association between calcium channels and migraines[5]. In addition, various studies have further confirmed that calcium and calcium-related proteins may play an important role in the process of migraine[6].

Meanwhile, detection of the changes in the concentration of extracellular calcium ions in this process will provide us with a method for studying nerve conduction[7]. It is reasonable to control the neuro-transmit process by changing the state of calcium in the inflammatory soup (IS) stimulation the sagittal sinus migraine model, and the pain signal is uploaded from the trigeminal meningeal branch, which eventually reaches the cortical sensory area and produces pain.

CaMPARI-GFP is an engineered fluorescent protein which enabled marking active neuronal populations with single-cell and subsecond resolution[8]. Viral expression of fluorescent tracers allows purely anterograde labeling. Following injection of AAV-hSyn-9-CaMPARI-GFP into the physiologically defined area in the VPM nucleus of the thalamus, labeling in the thalamus-cortex had a green appearance. Online electrochemical measurements of $\mathrm{Ca}^{2+}$ in rat brain is advantageous in terms of its simplicity both in instrumentation and in the experimental procedures and near real-time nature, and is thus highly anticipated to fifind wide applications in understanding of chemical events involved in some physiological and pathological processes[9]. This migraine process may be greatly documented by realtime monitoring of extracellular calcium dynamics. So far, the role of calcium antagonists in the prevention and treatment of migraine has been increasingly recognized[4]. The flunarizine, a non-selective calcium antagonist, has been shown in many controlled clinical studies to be effective in migraine prophylaxis[10]. Therefore, the purpose of this study is to explore whether VPM is related to pain transmission of migraine and to find whether flunarizine has a protective effect on VPM.

\section{Methods}

Part 1 Green fluorescence image of VPM-sensory cortex projection

Animals and virus particles. All experiments in this study adhered to the guidelines of the Committee for Research and Ethical Issues of IASP. All procedures were approved by the Chinese People's Liberation Army General Hospital animal experimental ethics committee. Adult healthy male Sprague-Dawley (SD) rats (6-10 weeks of age, weighing approximately 200-240 g, the Chinese People's Liberation Army General Hospital laboratory animal center) were used for virus injection into VPM. Virus particles for AAV-hSyn-9CaMPARI-GFP were obtained from Shanghai hang sheng material technology co. LTD (infective titer $1{ }^{\star} 10^{12}$ copies/mL). Adeno-associated virus AAV-hSyn-9 expressing CaMPARI-GFP was stereotaxically microinjected into both thalamic nuclei. CCD-camera epifluorescence microscopy and confocal laser scanning microscopy were used to quantify the area of VPM projection in the barrel field.

Surgery and stereotaxic injections. Rats were anesthetized with $3 \%$ pentobarbital sodium by intraperitoneal injection of $1.5 \mathrm{ml} / \mathrm{kg}$. The head of the rat was fixed on the stereolocator, and the scalp 
was cut layer by layer to expose the skull, showing sagittal fissure and anterior and posterior fontanelle. The anterior fontanelle point is taken as the coordinate zero point $(0,0,0)(\mathrm{mm})$, and the skull plane is adjusted to keep level. Dental drill on the skull respectively in $(2,1,0),(3.6,2.8,0),(3.6,2.8,0)(\mathrm{mm})$ drill three coordinate point $0.64 \mathrm{~mm}$ diameter hole. The three holes were respectively the catheterized sites of inflammatory decoction and the puncture injection sites of AAV-hSyn-9-CaMPARI-GFP virus in the VPM nuclei of the thalamus on both sides. Calibrated micropipettes were used to inject an estimated volume of $10 \mu \mathrm{L}$ the virus. The RWD stereotaxic alignment system was used with precise adjustments of the head in all 3 axes prior to injection[11]. A PE tube with a diameter of $0.64 \mathrm{~mm}$ was retained and fixed with epoxy resin. The skin was sutured layer by layer. The skin was recovered and fed normally for 2 weeks.

Grouping and drug interventions. Fifteen transfected rats were randomly divided into three groups: Flunarizine intervention group, Nimodipine intervention group and Normal saline sham group. Each rat was singly housed and had access to food and water ad libitum. At the $11^{\text {th }}, 12^{\text {th }}, 13^{\text {th }}$ day after the transfection model surgery, rats in each group were given flunarizine at $1 \mathrm{mg} / \mathrm{kg}$ (Sibelium Xian Janssen Pharmaceutical Ltd), nimodipine at $6 \mathrm{mg} / \mathrm{kg}$ (Nimotop Bayer Healthcare Ltd.) or normal saline.

Preparation of frozen brain slices. At the $14^{\text {th }}$ day after the surgery, after the last drug infusions, rats in each group were recorded on video for behavioral observation. Half an hour later, rats were stimulated with $20 \mu \mathrm{L}$ IS, and rats' behaviors were recorded and observed for 20 minutes. Rats were anesthetized with a lethal dose of $3 \%$ pentobarbital and transcardially perfused with $0.1 \mathrm{M}$ phosphate-buffered solution (PBS). The brain was extracted and embedded in Optimal Cutting Temperature (OCT) Compound (SAKURA), then incubated overnight at $-20^{\circ} \mathrm{C}$. Tangential sections with $20 \mu \mathrm{m}$ thickness were prepared.

Fluorescence microscopy. Epifluorescence images were obtained using an Olympus BX-43 upright microscope equipped with GFP (excitation $488 \mathrm{~nm}$, emission $520 \mathrm{~nm}$ ) fluorescence filter sets. VPM and sensory cortex (S) area measurements were performed using the analysis program package (Olympus) on images taken at $\times 4$ magnification.

Part 2 Extracellular calcium ion dynamics of VPM

Animals and drugs

Rats were housed (1-3 per cage, same sex) in the animal facility of the institute of Chinese Academy of Science key laboratory of analytical chemistry for living Biosystems (ACLCAS) on a 12-HR light/dark cycle with constant temperature $\left(23-26^{\circ} \mathrm{C}\right)$, humidity $(30-45 \%)$ as well as food and water ad libitum. Adult 
SD rats (8-12 weeks old, Male, weight 250-300g, provided by the Chinese People's Liberation Army General Hospital laboratory animal center) were used in the experiments.

\section{Surgery}

Calcium ion selective Carbon fiber microelectrodes ( $\mathrm{Ca}^{2+} \mathrm{CFMEs}$ ) are provided by the institute of Chinese Academy of Science Key Laboratory of Analytical Chemistry for Living Biosystems (ACLCAS). Each ion selective electrode was calibrated before the experiment. The electrode exhibited stable and rapid responses when it is introduced to various concentrations of $\mathrm{Ca}^{2+}$. The electrode exhibited near Nernstian responses with detection limits of less than $10^{-1} \mathrm{M}$, a linear response range between $10^{-8}-10^{-1} \mathrm{M}$, and a temporal resolution of $25 \mathrm{~ms}$. After inducing anesthesia, the rats were quickly fixed to the stereotaxy, and provided sustained isoflurane gas anesthesia (2.0-2.5L / min, and oxygen flow rate $0.5-0.7 \mathrm{~L} / \mathrm{min})$. The fur was removed carefully to fully expose the skull with a diameter of $1 \mathrm{~cm}$. The subcutaneous tissue and the periosteum were then separated to expose the anterior fontanelle. The posterior fontanel was used as a reference point, with a dental drill $(-2,-1,0),(-3.6,2.8,0),(-3.6,-2.8,0)(\mathrm{mm})$. The diameters of the drilling/opening windows were $0.64 \mathrm{~mm}, 5 \mathrm{~mm}$ and $0.64 \mathrm{~mm}$, respectively, at the injection point of the inflammation soup, the electrode ( $\mathrm{Ca}^{2+} \mathrm{CFMEs}$ ) placement point and the reference electrode placement point. In $(-2,-1,0)(\mathrm{mm})$, the injection point of the inflammatory soup was implanted into the diameter of $0.64 \mathrm{~mm}$ PE tube (the PE tube enters the skull, do not oppress the brain tissue), and the PE tube was closed and fixed with epoxy resin. After the recording electrode was placed in the window $(-3.6,2.8,0)$ $(\mathrm{mm})$, the dura mater should be carefully selected with syringe needle, and the brain tissue should be avoided as far as possible. $\mathrm{Ca}^{2+} \mathrm{CFMEs}$ and reference electrode were calibrated before the experiment. The electrode was placed at $(-3.6,2.8,6.1)(\mathrm{mm})$ by using the stereo locator, and the reference electrode was placed at $(-3.6,2.8,0)(\mathrm{mm})$. Artificial cerebro-spinal fluid (aCSF) was applied to protect the exposed brain tissue, and the electrochemical workstation was used to record Calcium voltage. Continuous recording started a smooth baseline record, which continued for 900 s after injection of $20 \mu \mathrm{L}$ inflammation soup (histamine and serotonin, bradykinin mixture of $2 \mathrm{~mm}$ and $0.2 \mathrm{~mm}$ prostaglandin E2 dissolve in normal saline区pH=5.5).

Grouping and drug intervention

Twenty SD male rats were divided into four groups according to the random number method: Single stimulus group, Double stimuli group, Flunarizine intervention group, Nimodipine intervention group. The drug intervention group (Flunarizine, Nimodipine) was administered three days before the operation, and the drug was administered half an hour before the experiment. The single stimulus group and the drug intervention group were placed on the electrode and recorded according to the procedure. Before IS stimulation, $20 \otimes \mathrm{L}$ of normal saline was injected to detect the patience of the injection tube and the reactivity of the sagittal sinus meningioma in the same amount of liquid. At least 1 hour after the injection of inflammatory soup in each group of rats, the secondary stimulation group was injected into the inflammatory soup after the first stimulation for 1 hour. 
Nimodipine $6 \mathrm{mg} / \mathrm{kg}$ (Nimotop Bayer Healthcare Ltd.) and Flunarizine $1 \mathrm{mg} / \mathrm{kg}$ (Sibelium Xian Janssen Pharmaceutical Ltd.) were given to the experimental rats via nasogastric tube daily at 8-10am 3 days prior to the experimental day, and given again on the day of experiment in half an hour. At the end of the experiment, the rats were given an intraperitoneal injection of $3 \%$ of the lethal dose of pentobarbital.

\section{Data analysis}

OriginPro $2017 \mathrm{C}$ software was used for data sorting, and SPSS 22.0 software was used for statistical analysis. The baseline value was the pre-stimulus concentration of inflammatory soup, and was defined as the initial value; the calcium concentration increased to the maximum after the inflammatory soup was stimulated was defined as the maximum. The slope of the fitting line with logarithmic linear decline after the stimulation of the inflammatory soup was defined as the slope. The time defined as peak time was defined as the time of the increase of calcium ion concentration from the inflammatory soup to the maximum. Recovery time was defined as the time from stimulus to recovery starting concentration. Quantitative data was used to mean the standard deviation of the mean, and the $t$-test of quantitative data was used in the group.

\section{Results}

Part 1 Green fluorescence image of VPM-sensory cortex projection

VPM axons arising from bundles in the stria enter the subcortical white matter (SCWM) and bend into a tangential direction almost parallel to the cortical surface. VPM projections to cortical fibers were observed in different layers of vertical slices. In this study, the VPM nucleus of the thalamus, lateral horn of the hippocampus (HC-CA3), lateral paraventricular (LV), and sensory cortex (S1) were observed (Figures 1). Fluorescence imaging showed bilateral VPM green fluorescence imaging in 5 rats in flunarizine intervention group, while 3 out of 5 rats in nimodipine intervention group showed left VPM fluorescence imaging, and the other 2 rats showed bilateral VPM fluorescence imaging. In normal saline sham group, 3 out of 5 rats showed left unilateral calcium fluorescence imaging and 2 rats showed bilateral fluorescence imaging.

Calcium ion green fluorescent image of the sensory cortex was performed. Droplet fluorescence in S1 of the sensory cortex was observed in 2 rats in normal saline sham group and 1 rat in nimodipine intervention group. Fluorescence imaging of the fiber bundles near the hippocampus and the lateral ventricle showed fluorescence imaging of the left lateral ventricle and the left lateral ventricle of 5 rats in normal saline sham group. In nimodipine intervention group, fluorescence imaging was observed in the left hippocampus of 5 rats and the lateral ventricle of 2 rats. Fluorescence imaging was performed on the left hippocampus of only 1 out of 5 rats in flunarizine intervention group (Table 1).

Part 2 Extracellular calcium ion dynamics of VPM

2.1 Electrode calibration 
The $\mathrm{Ca}^{2+}$ CFME electrode was calibrated in a standard concentration of calcium ions solution before the beginning of the experiment (Figure 2), and its reactivity was tested. We calculated the electrode's Voltage-Concentration parameter $(a, b)$ with a simplified version (voltage-concentration equation) of the Nernst equation.

$$
E_{C a^{2+}}=b+a \log \left(C a^{2+}\right)(a=\text { Slope }, b=\text { Intercept })
$$

\subsection{Stimulus}

Single stimulus group

A pain signal was produced due to physical traction. Before IS stimulation, calcium voltage change was observed after the same amount of saline stimulation, and no significant change was observed after 300 seconds of continuous observation. When the inflammatory soup was stimulated, the concentration of calcium ions around the VPM nuclear showed an increase and then a decrease, and the overall logarithmic linear declined (Figure 3 ). The mean of the initial concentration was $1.31 \times 10^{-3} \pm 1.51 \times 10^{-}$ ${ }^{3} \mathrm{~mol} / \mathrm{L}$, the peak concentration was $2.25 \times 10^{-3} \pm 2.36 \times 10^{-3} \mathrm{~mol} / \mathrm{L}$, and peak/initial was $2.59 \pm 1.59$, with a slope of $\left(-3.12 \times 10^{-4} \pm 3.24 \times 10^{-4}\right)$. The time of 0 -max was $491.6 \pm 325.9 \mathrm{~s}$, with max-recovery of $777.4 \pm 737.6 \mathrm{~s}$. There was no significant change in the real time electrochemical monitoring in the

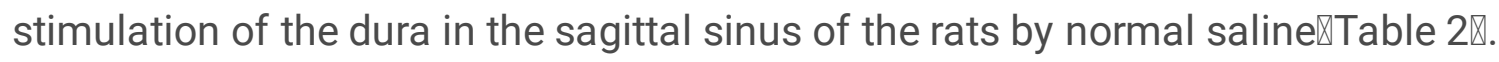

Double stimuli group

Comparing to the single stimulus, the secondary stimulus presented similar results, which was an increase following by a decrease in voltage (Figure 4). The mean of the initial concentration of the first stimulus was $1.98 \times 10^{-4} \pm 2.05 \times 10^{-4} \mathrm{~mol} / \mathrm{L}$, the peak concentration was $3.51 \times 10^{-4} \pm 2.16 \times 10^{-4} \mathrm{~mol} / \mathrm{L}$, and peak/initial was $2.48 \pm 1.57$, with a slope of $\left(-1.05 \times 10^{-4} \pm 1.53 \times 10^{-5}\right)$. The time of 0 -max was $274.4 \pm 191.2 \mathrm{~s}$, with a max-recovery of $572.2 \pm 446.3 \mathrm{~s}$. The mean of the secondary concentration of the first stimulus was $1.12 \times 10^{-4} \pm 1.26 \times 10^{-4} \mathrm{~mol} / \mathrm{L}$, the peak concentration was $2.64 \times 10^{-4} \pm 1.68 \times 10^{-4} \mathrm{~mol} / \mathrm{L}$, and peak/initial was $3.34 \pm 2.52$, with a slope of $\left(-2.09 \times 10^{-4} \pm 1.18 \times 10^{-5}\right)$. The time of 0 -max was $688.2 \pm 484.7 \mathrm{~s}$, with a maxrecovery of $404.8 \pm 306.2 \mathrm{~s} \otimes T$ Table $2 \bigotimes$. In contrast to the first stimulus, paired-samples $t$ test of the second stimulus showed no obvious difference in the ratio of the Peak and Initial, namely the increase amplitude ratio $\triangle t=-1.47, v=4, p=0.2270 .05 \rrbracket$. The time of $0-\max (t=-1.88, v=4, p=0.1370 .05)$ and recovery $(t=-1.59, v=$ $4, p=0.1900 .05)$ also showed no obvious difference. There was no significant difference between the two stimuli of slope $(t=1.81, v=4, p=0.15 \square 0.05) \otimes T a b l e 3 \otimes$.

2.3 Intervention

Nimodipine intervention group 
Nimodipine. Similar to the single-stimulus group, the nimodipine intervention group exhibited an increase in extracellular $\mathrm{Ca}^{2+}$ concentration followed by a decrease (Figure 5 ). The mean of the initial concentration was $3.64 \times 10^{-4} \pm 3.97 \times 10^{-4} \mathrm{~mol} / \mathrm{L}$, the peak concentration was $-5.84 \times 10^{-4} \pm 3.02 \times 10^{-4} \mathrm{~mol} / \mathrm{L}$, and the peak/initial was $1.39 \pm 0.32$, with a slope of $-5.84 \times 10^{-4} \pm 3.02 \times 10^{-4}$. The time of 0 -max was $137.8 \pm 167.1 \mathrm{~s}$, and the max-recovery was $264.0 \pm 145.8 \mathrm{~s}$ (Table 2). There was no significant difference in the slope between the nimodipine intervention group and the single stimulation group $₫ d f=8, t=1.379$, $p=0.205 \rrbracket$ (Table 4), suggesting that the change trend of calcium ion concentration in the two groups after stimulation was similar.

Flunarizine intervention group

Flunarizine. Significantly different from the single-stimulus group, double-stimuli group and the nimodipine intervention group, the flunarizine intervention group did not show a decrease or an increase in calcium ion concentration (Figure 5). Flunarizine was given prior to IS stimulation, and the changes observed in VPM extracellular $\mathrm{Ca}^{2+}$ concentration were significantly different from those in the other three groups. The mean of the initial concentration was $7.54 \times 10^{-5} \pm 7.08 \times 10^{-5} \mathrm{~mol} / \mathrm{L}$, the peak concentration was $2.45 \times 10^{-4} \pm 2.51 \times 10^{-4} \mathrm{~mol} / \mathrm{L}$, and the peak/initial was $3.07 \pm 2.39$. The slope was $9.07 \times 10^{-5} \pm 6.03 \times 10^{-5}$ (Table 2). The change trend of calcium ion concentration in the intervention group was significantly different from that in the single stimulus group, and the slope was significantly different $(d f=8, t=-2.729$, $p=0.026)$. At the same time, there were significant differences between the flunarizine intervention group and nimodipine intervention group $(d f=8, t=-4.904, p=0.001)$. (Table 4)

\section{Discussion}

The normal human brain is an orderly set of chemical reaction. Migraine is a common neurovascular disease and some studies suggest that it may be related to ion channels[5]. Familial hemiplegic migraine (FHM), a rare autosomal dominant subtype of migraine with aura, is the first evidence implicating migraine to be a neuronal channelopathy[12]. FHM1 mutations are usually missense and gain-offunction, leading to increased $\mathrm{Ca}^{2+}$ influx, which has the effect of enhancing glutamatergic neurotransmission[6]. Flunarizine, a nonspecific calcium antagonist, is well documented for prophylactic treatment of migraine[13;14;15]. Several studies of nimodipine in preventing migraines have failed to show that it is better than placebo[16;17;18]. This study intends to explore by detecting the VPM nuclear extracellular calcium of alive migraine animal model headache process in real-time monitoring. The two calcium antagonists which can traverse blood brain barrier always used for the prevention and treatment of migraine were used as a means of intervention, further verify the method reliability and explore the migraine and intervention mechanism.

Previous studies have demonstrated that VPM neurons in thalamus participate in headache signal transmission through electrophysiological means, and are an important relay nucleus for headache sensation[19]. Calcium GFP imaging once again confirmed the role of the VPM nucleus in headache signaling. At the same time, fluorescence imaging of VPM nucleus was also found in nimodipine 
intervention group and flunarizine intervention group, suggesting that thalamic neurons were still activated after the intervention. Real-time VPM extracellular calcium ion monitoring IS stimulation reaction showed the first increase in all groups, suggesting that VPM is involved in the transmission of head and face pain signals.

Nimodipine is a dihydropyridine-derivative calcium-channel blocking agent that affects the CNS preferentially[16]. Flunarizine is a non - selective non - dihydropyridine calcium ion antagonist and can pass through the blood-cerebro-spinal fluid barrier[20]. A large number of clinical studies have confirmed that nimodipine is not effective in the treatment and prevention of migraine[15;17; 18; 21]. The efficacy of flunarizine in the prevention and treatment of migraine is also clinically proven and recommended for clinical application[10]. GFP fluorescence imaging of the sensory cortex suggested that pain signals were transmitted to the sensory cortex. Fluorescence imaging of the sensory cortex was observed in the normal saline sham group and the nimodipine intervention group, while no fluorescence imaging was observed in the sensory cortex in flunarizine intervention group.

Migraine is the headache of a hyperactive calcium channel[5]. Calcium channel antagonists have been adopted in migraine treatment for many years, and flunarizine has been shown to be effective. Other calcium channel antagonists that can cross the blood-brain barrier lack evidence. However, the mechanism of its anti-migraine action remains unclear. Studies on single-celled neurons with extracorporal patch-clamp calcium channel confirmed that flunarizine could block the process of calcium intracellular flow, and speculated that it might act on the secondary release process of calcium intracellular flow in central neurons. Present study showed that extracellular calcium ion concentration remained almost unchanged after flunarizine intervention, suggesting that net calcium influx may be blocked and thus play an anti-migraine role.

In this study, the extra-cellular calcium dynamics of VPM nucleus was good in monitoring the transmission of pain signals from the superior sagittal sinus to the hypothalamus after transposition. As the Single and the Double stimuli group, the concentration of extracellular calcium ions decreased when given the stimulus. The decrease curve of extracellular calcium concentration in neurons is logarithmic linear and slope is $-1.05 \times 10^{-4} \pm 1.44 \times 10^{-5} / \mathrm{s}$ (The mean and mean difference of slope between the single stimulus group and the second stimulus group after the first stimulus). It is similar to logarithmic linearity when neurotransmitter quantization is released. At the same time, the rate of decline of calcium ions is approximately the same as the amount of calcium ions required for each transmitter quantum at $10^{-5}$ to $10^{-6}[22]$. Comparing to the change of calcium concentration after the stimulation of IS, the use of physiological saline before IS has no response. Furthermore, the second stimulation group showed similar performance to the first stimulation after repeated IS stimulation. According to the trend of calcium ions $₫$ consistent with the clinical intervention effect, the extra-cellular calcium dynamics performance after nimodipine intervention was similar to that of the sham intervention group, and the flunarizine intervention group was significantly different from the nimodipine intervention group and the sham intervention group. 


\section{Conclusions}

In conclusion, VPM might play an important role in pain signal transmission of migraine and Flunarizine as an effective preventive drug in clinical application can alleviate such $\mathrm{Ca}^{2+}$ concentration in VPM neurons to exert its anti-migraine effects.

\section{Declarations}

\section{Acknowledgment}

Not applicable.

\section{Funding}

The authors were supported financially by the National Natural Science Foundation Item of China (81771180), Natural Science Foundation of Beijing Municipality (7162178).

\section{Availability of data and materials}

The datasets analyzed during the current study are available from the corresponding author on reasonable request.

\section{Author contribution}

RL, LM, SY supervised the experiment design. WG, LZ conceived the study. W $G$ and $L Z$ performed most of the experiments, analyzed the data, and wrote the manuscript. $\mathrm{CH}, \mathrm{QZ}, \mathrm{YW}$ revised the manuscript. All of the authors read and approved the final manuscript. All authors read and approved the final manuscript.

\section{Ethics approval and consent to participate}

All experiments in this study adhered to the guidelines of the Committee for Research and Ethical Issues of IASP. Experimental procedures and protocols were approved by the Chinese People's Liberation Army General Hospital animal experimental ethics committee.

\section{Consent for publication}

Not applicable. 


\section{Competing interests}

The author(s) declared no potential conflicts of interest with respect to the research, authorship, and/or publication of this article.

\section{Abbreviations}

VPM ventralis posteromedialis

$\mathrm{Ca}^{2+}$ Calcium ions

GFP Green fluorescence protein

PBS phosphate-buffered solution

$\mathrm{Ca}^{2+}$ CFMEs Calcium ion selective Carbon fiber microelectrodes

aCSF artificial cerebro-spinal fluid

LV lateral paraventricular

FHM Familial hemiplegic migraine

CNS Central Nervous System

\section{References}

[1] B. R, N. R, and B. D, Migraine: multiple processes, complex pathophysiology. The Journal of neuroscience : the official journal of the Society for Neuroscience 35 (2015) 6619-29.

[2] B. L, B. J, B. G, K. V, B. R, and B. D, Brain network alterations in the inflammatory soup animal model of migraine. Brain research 1660 (2017) 36-46.

[3] K. B, and M. R, A study of synaptic transmission in the absence of nerve impulses. The Journal of physiology 192 (1967) 407-36.

[4] R. MB, and D. A, Sporadic and familial hemiplegic migraine: pathophysiological mechanisms, clinical characteristics, diagnosis, and management. The Lancet. Neurology 10 (2011) 457-70.

[5] X. M, and R. C, The headache of a hyperactive calcium channel. Neuron 61 (2009) 653-4.

[6] A. CL, S. S, H. LM, and G. LR, Ion channelopathies and migraine pathogenesis. Molecular genetics and genomics : MGG 292 (2017) 729-739. 
[7] T. E, and N. K, Modulators of voltage-dependent calcium channels for the treatment of nervous system diseases. Recent patents on CNS drug discovery 4 (2009) 96-111.

[8] M. B, H. G, M. R, P.-A. A, F. BC, T. NF, L. J, Z. TA, D. A, P. D, B. TA, S. RNS, E. BJ, L. ME, T. GG, D. H, G. CE, O. TG, H. BT, and S. ER, Improved methods for marking active neuron populations. Nature communications 9 (2018) 4440.

[9] Z. Z, Z. L, L. Y, Y. P, and M. L, Online electrochemical measurements of Ca2+ and Mg2+ in rat brain based on divalent cation enhancement toward electrocatalytic NADH oxidation. Analytical chemistry 82 (2010) 9885-91.

[10] E. S, A. J, F. A, G. PJ, L. M, M. A, and S. PS, EFNS guideline on the drug treatment of migraine-revised report of an EFNS task force. European journal of neurology 16 (2009) 968-81.

[11] W. VC, B. RM, d.K. CP, K. T, and S. B, Dimensions of a projection column and architecture of VPM and POm axons in rat vibrissal cortex. Cerebral cortex (New York, N.Y. : 1991) 20 (2010) 2265-76.

[12] T. A, C. R, F. A, V. D, S. M, S. M, v.d.M. AM, F. MD, and P. D, Enhanced excitatory transmission at cortical synapses as the basis for facilitated spreading depression in $\mathrm{Ca}(\mathrm{v}) 2.1$ knockin migraine mice. Neuron 61 (2009) 762-73.

[13] L. M, G. L, L.M. L, and B. G, Flunarizine in migraine: a minireview. Headache 31 (1991) 388-91.

[14] S. SD, H. S, F. F, D. DW, A. C, and A. E, Evidence-based guideline update: pharmacologic treatment for episodic migraine prevention in adults: report of the Quality Standards Subcommittee of the American Academy of Neurology and the American Headache Society. Neurology 78 (2012) 1337-45.

[15] T. I, D.C. D, B. B, S. S, and B. PA, The pharmacological treatment of migraine in children and adolescents: an overview. Expert review of neurotherapeutics 12 (2012) 1133-42.

[16] L. MS, and S. EM, Nimodipine. A review of its pharmacodynamic and pharmacokinetic properties, and therapeutic potential in cerebrovascular disease. Drugs 37 (1989) 669-99.

[17] B. G, B. S, D.A. G, C. A, F. F, C. L, F.M. F, and B. A, Nimodipine versus flunarizine in common migraine: a controlled pilot trial. Headache 27 (1987) 76-9.

[18] N. A, L. C, P. N, D.A. G, R. G, and B. U, Long-term follow-up after flunarizine or nimodipine discontinuation in migraine patients. Cephalalgia : an international journal of headache 16 (1996) 33740 .

[19] B. R, J. M, G.-N. E, K. V, B. Z, H. R, B. L, and B. D, Thalamic sensitization transforms localized pain into widespread allodynia. Annals of neurology 68 (2010) 81-91. 
[20] T. K, and A. N, Calcium antagonist effects on low-threshold (T-type) calcium current in rat isolated hippocampal CA1 pyramidal neurons. The Journal of pharmacology and experimental therapeutics 256 (1991) 169-75.

[21] A. K, O. T, and T. N, Flunarizine, an anti-migraine agent, impairs nitroxidergic nerve function in cerebral arteries. European journal of pharmacology 329 (1997) 49-53.

[22] M. R, Transmitter release induced by injection of calcium ions into nerve terminals. Proceedings of the Royal Society of London. Series B, Biological sciences 183 (1973) 421-5.

\section{Tables}

Table 1. Distribution of calcium fluorescence protein imaging sites in the brain sections of rats in each group

\begin{tabular}{|c|c|c|c|c|c|c|c|c|c|}
\hline \multirow[t]{2}{*}{ Group } & \multirow[t]{2}{*}{ Number } & \multicolumn{2}{|c|}{ VPM } & \multicolumn{2}{|c|}{ HC-CA3 } & \multicolumn{2}{|c|}{ LV } & \multicolumn{2}{|c|}{$\mathrm{S} 1$} \\
\hline & & $\mathrm{L}$ & $\mathrm{R}$ & $\mathrm{L}$ & $\mathrm{R}$ & $\mathrm{L}$ & $\mathrm{R}$ & $\mathrm{L}$ & $\mathrm{R}$ \\
\hline \multirow[t]{5}{*}{ Sham } & 1 & $\sqrt{ }$ & & $\sqrt{ }$ & & $\sqrt{ }$ & & & \\
\hline & 2 & $\sqrt{ }$ & & $\sqrt{ }$ & & & & & \\
\hline & 3 & $\sqrt{ }$ & $\sqrt{ }$ & $\sqrt{ }$ & & $\sqrt{ }$ & & $\sqrt{ }$ & \\
\hline & 4 & $\sqrt{ }$ & & $\sqrt{ }$ & & & & & \\
\hline & 5 & $\sqrt{ }$ & $\sqrt{ }$ & $\sqrt{ }$ & & $\sqrt{ }$ & & $\sqrt{ }$ & \\
\hline \multirow[t]{5}{*}{ Flunarizine } & 1 & $\sqrt{ }$ & $\sqrt{ }$ & & & & & & \\
\hline & 2 & $\sqrt{ }$ & $\sqrt{ }$ & $\sqrt{ }$ & & & & & \\
\hline & 3 & $\sqrt{ }$ & $\sqrt{ }$ & & & & & & \\
\hline & 4 & $\sqrt{ }$ & $\sqrt{ }$ & & & & & & \\
\hline & 5 & $\sqrt{ }$ & $\sqrt{ }$ & & & & & & \\
\hline \multirow[t]{5}{*}{ Nimodipine } & 1 & $\sqrt{ }$ & & $\sqrt{ }$ & & & & & \\
\hline & 2 & $\sqrt{ }$ & $\sqrt{ }$ & $\sqrt{ }$ & & $\sqrt{ }$ & & $\sqrt{ }$ & \\
\hline & 3 & $\sqrt{ }$ & $\sqrt{ }$ & $\sqrt{ }$ & & & & & \\
\hline & 4 & $\sqrt{ }$ & & $\sqrt{ }$ & & & & & \\
\hline & 5 & $\sqrt{ }$ & & $\sqrt{ }$ & & $\sqrt{ }$ & & & \\
\hline
\end{tabular}

Table 2. Characteristics of calcium ion concentration in each group 


\begin{tabular}{|c|c|c|c|c|c|c|}
\hline Group & Parameter & Number & Minimum & Maximum & Mean & Standard deviation \\
\hline \multirow[t]{6}{*}{ Single-stimulus group } & Initial (M) & 5 & $1.05 \mathrm{E}-4$ & $3.47 \mathrm{E}-3$ & $1.31 \mathrm{E}-3$ & $1.51 \mathrm{E}-3$ \\
\hline & Peak (M) & 5 & $5.19 \mathrm{E}-4$ & $5.13 \mathrm{E}-3$ & $2.25 \mathrm{E}-3$ & $2.36 \mathrm{E}-3$ \\
\hline & Peak : initial & 5 & 1.48 & 4.93 & 2.59 & 1.39 \\
\hline & Slope & 5 & $-8.43 E-4$ & $-4.03 E-7$ & $-3.12 \mathrm{E}-4$ & $3.24 \mathrm{E}-4$ \\
\hline & Maximum & 5 & 75.00 & 839.00 & 491.60 & 325.99 \\
\hline & Recovery (s) & 5 & 143.00 & 2026.00 & 777.40 & 737.61 \\
\hline \multirow[t]{12}{*}{ Double-stimulus group } & I1 (M) & 5 & $6.44 \mathrm{E}-5$ & $5.55 \mathrm{E}-4$ & $1.98 \mathrm{E}-4$ & $2.06 \mathrm{E}-4$ \\
\hline & P1 (M) & 5 & $1.09 \mathrm{E}-4$ & $6.90 \mathrm{E}-4$ & $3.52 \mathrm{E}-4$ & $2.17 \mathrm{E}-4$ \\
\hline & $\mathrm{P} 1: \mathrm{I} 1$ & 5 & 1.24 & 4.54 & 2.48 & 1.57 \\
\hline & S1 & 5 & $-1.23 \mathrm{E}-4$ & $-8.21 \mathrm{E}-5$ & $-1.05 E-4$ & $1.53 \mathrm{E}-5$ \\
\hline & M1 & 5 & 60.00 & 543.00 & 274.40 & 191.24 \\
\hline & $\mathrm{R} 1$ (s) & 5 & 263.00 & 1332.00 & 572.20 & 446.30 \\
\hline & I2 & 5 & $1.20 \mathrm{E}-5$ & $3.27 \mathrm{E}-4$ & $1.12 \mathrm{E}-4$ & $1.26 \mathrm{E}-4$ \\
\hline & $\mathrm{P} 2$ & 5 & 1.92E-5 & $4.56 \mathrm{E}-4$ & $2.64 \mathrm{E}-4$ & $1.68 \mathrm{E}-4$ \\
\hline & $\mathrm{P} 2: \mathrm{I} 2$ & 5 & 1.39 & 7.00 & 3.34 & 2.52 \\
\hline & $\mathrm{S} 2$ & 5 & $-3.90 \mathrm{E}-4$ & $-1.19 \mathrm{E}-4$ & $-2.09 E-4$ & $1.19 \mathrm{E}-4$ \\
\hline & M2 & 5 & 70.00 & 848.00 & 404.80 & 306.19 \\
\hline & $\mathrm{R} 2$ & 5 & 268.00 & 1380.00 & 688.20 & 484.76 \\
\hline \multirow[t]{6}{*}{ Nimodipine intervention group } & Initial (M) & 5 & $2.78 \mathrm{E}-5$ & $9.51 \mathrm{E}-4$ & $3.64 \mathrm{E}-4$ & $3.98 \mathrm{E}-4$ \\
\hline & Peak (M) & 5 & $3.31 \mathrm{E}-5$ & $1.76 \mathrm{E}-3$ & $6.04 \mathrm{E}-4$ & $7.45 \mathrm{E}-4$ \\
\hline & Peak : initial & 5 & 1.13 & 1.85 & 1.39 & .32 \\
\hline & Slope & 5 & $-8.74 \mathrm{E}-4$ & $-1.35 \mathrm{E}-4$ & $-5.85 E-4$ & 3.02E-4 \\
\hline & Maximum & 5 & 25.00 & 432.00 & 137.80 & 167.11 \\
\hline & Recovery (s) & 5 & 65.00 & 402.00 & 264.00 & 145.87 \\
\hline \multirow[t]{4}{*}{ Flunarizine intervention group } & Initial (M) & 5 & $1.14 \mathrm{E}-5$ & $1.83 \mathrm{E}-4$ & $7.55 \mathrm{E}-5$ & $7.08 \mathrm{E}-5$ \\
\hline & Peak (M) & 5 & $1.21 \mathrm{E}-5$ & $6.33 \mathrm{E}-4$ & $2.45 \mathrm{E}-4$ & $2.51 \mathrm{E}-4$ \\
\hline & Peak : initial & 5 & 1.06 & 6.91 & 3.08 & 2.40 \\
\hline & Slope & 5 & $7.50 \mathrm{E}-6$ & $1.63 \mathrm{E}-4$ & 9.07E-5 & 6.03E-5 \\
\hline
\end{tabular}

I1, P1, C1, S1, M1, and R1 represent the initial concentration, peak concentration, peak/initial concentration ratio, slope, and recovery time of the first stimulus, respectively. I2, P2, C2, S2, M2, R2 represent the corresponding parameters of the second stimulus.

Table 3. Paired-sample t-test of parameters of the two stimuli 


\begin{tabular}{|c|c|c|c|c|c|c|c|c|}
\hline & \multicolumn{5}{|c|}{ Pairwise difference } & \multirow[t]{3}{*}{$\mathrm{t}$} & \multirow[t]{3}{*}{ df } & \multirow{3}{*}{$\begin{array}{l}\text { Sig. (2- } \\
\text { tailed) }\end{array}$} \\
\hline & \multirow[t]{2}{*}{ Mean } & \multirow[t]{2}{*}{$\begin{array}{l}\text { Std. } \\
\text { deviation }\end{array}$} & \multirow[t]{2}{*}{$\begin{array}{l}\text { Std. error of the } \\
\text { mean }\end{array}$} & \multicolumn{2}{|c|}{$\begin{array}{l}\text { 95\% confidence interval of the } \\
\text { difference }\end{array}$} & & & \\
\hline & & & & Lower & Upper & & & \\
\hline I1 - I2 & $\begin{array}{l}8.62 \mathrm{E}- \\
5\end{array}$ & 8.34E-5 & $3.73 E-5$ & $-1.73 E-5$ & $1.90 \mathrm{E}-4$ & 2.31 & 4 & .082 \\
\hline $\mathrm{P} 1$ - P2 & $\begin{array}{l}8.83 \mathrm{E}- \\
5\end{array}$ & 8.53E-5 & $3.82 \mathrm{E}-5$ & $-1.76 \mathrm{E}-5$ & $1.94 \mathrm{E}-4$ & 2.32 & 4 & .082 \\
\hline C1 - C2 & -0.87 & 1.32 & 0.59 & -2.50 & 0.77 & -1.47 & 4 & .216 \\
\hline $\mathrm{S} 1-\mathrm{S} 2$ & $\begin{array}{l}1.04 \mathrm{E}- \\
4\end{array}$ & $1.28 \mathrm{E}-4$ & $5.74 \mathrm{E}-5$ & $-5.57 \mathrm{E}-5$ & $2.63 \mathrm{E}-4$ & 1.81 & 4 & .145 \\
\hline $\begin{array}{l}\text { M1 - } \\
\text { M2 }\end{array}$ & -130.40 & 155.14 & 69.38 & -323.03 & 62.23 & -1.88 & 4 & .133 \\
\hline R1 - R2 & -116.00 & 163.18 & 72.98 & -318.62 & 86.62 & -1.59 & 4 & .187 \\
\hline
\end{tabular}

I1, P1, C1, S1, M1, and R1 represent the initial concentration, peak concentration, peak/initial concentration ratio, slope, and recovery time of the first stimulus, respectively. I2, P2, C2, S2, M2, R2 represent the corresponding parameters of the second stimulus.

Table 4. Independent-samples t-tests comparing the slopes of different groups

\section{Levene's test for t-test for equality of means} equality of variances

F Sig. $t \quad$ df Sig. Mean Std. error of $95 \%$ confidence (2- difference the interval of the tailed) difference difference

\begin{tabular}{|c|c|c|c|c|c|c|c|c|c|c|}
\hline & & & & & & & & & Lower & Upper \\
\hline \multirow{2}{*}{$\begin{array}{l}\text { S1- } \\
\text { S4 }\end{array}$} & $\begin{array}{l}\text { Equal variances } \\
\text { assumed }\end{array}$ & 4.489 & .067 & -2.729 & 8 & .026 & $-4.02 \mathrm{E}-4$ & $1.47 \mathrm{E}-4$ & $-7.42 \mathrm{E}-4$ & $-6.24 \mathrm{E}-5$ \\
\hline & $\begin{array}{l}\text { Equal variances } \\
\text { not assumed }\end{array}$ & & & -2.729 & 4.277 & .049 & $-4.02 \mathrm{E}-4$ & $1.47 \mathrm{E}-4$ & $-8.01 E-4$ & $-3.24 \mathrm{E}-6$ \\
\hline \multirow[t]{2}{*}{$\begin{array}{l}\mathrm{S} 2- \\
\mathrm{S} 4\end{array}$} & $\begin{array}{l}\text { Equal variances } \\
\text { assumed }\end{array}$ & 7.444 & .026 & -7.046 & 8 & .000 & $-1.96 \mathrm{E}-4$ & $2.78 E-5$ & $-2.60 \mathrm{E}-4$ & $-1.32 \mathrm{E}-4$ \\
\hline & $\begin{array}{l}\text { Equal variances } \\
\text { not assumed }\end{array}$ & & & -7.046 & 4.510 & .001 & $-1.96 \mathrm{E}-4$ & $2.78 \mathrm{E}-5$ & $-2.70 \mathrm{E}-4$ & $-1.22 \mathrm{E}-4$ \\
\hline \multirow{2}{*}{$\begin{array}{l}\text { S3- } \\
\text { S4 }\end{array}$} & $\begin{array}{l}\text { Equal variances } \\
\text { assumed }\end{array}$ & 10.026 & .013 & -4.904 & 8 & .001 & $-6.76 \mathrm{E}-4$ & $1.38 \mathrm{E}-4$ & $-9.93 E-4$ & $-3.58 E-4$ \\
\hline & $\begin{array}{l}\text { Equal variances } \\
\text { not assumed }\end{array}$ & & & -4.904 & 4.318 & .007 & $-6.76 \mathrm{E}-4$ & $1.38 \mathrm{E}-4$ & $-1.05 E-3$ & $-3.04 \mathrm{E}-4$ \\
\hline \multirow[t]{2}{*}{$\begin{array}{l}\text { S1- } \\
\text { S3 }\end{array}$} & $\begin{array}{l}\text { Equal variances } \\
\text { assumed }\end{array}$ & .008 & .931 & 1.379 & 8 & .205 & $2.73 E-4$ & $1.98 \mathrm{E}-4$ & $-1.84 \mathrm{E}-4$ & 7.30E-4 \\
\hline & $\begin{array}{l}\text { Equal variances } \\
\text { not assumed }\end{array}$ & & & 1.379 & 7.961 & .205 & $2.73 \mathrm{E}-4$ & $1.98 \mathrm{E}-4$ & $-1.84 \mathrm{E}-4$ & 7.31E-4 \\
\hline
\end{tabular}

\section{Figures}



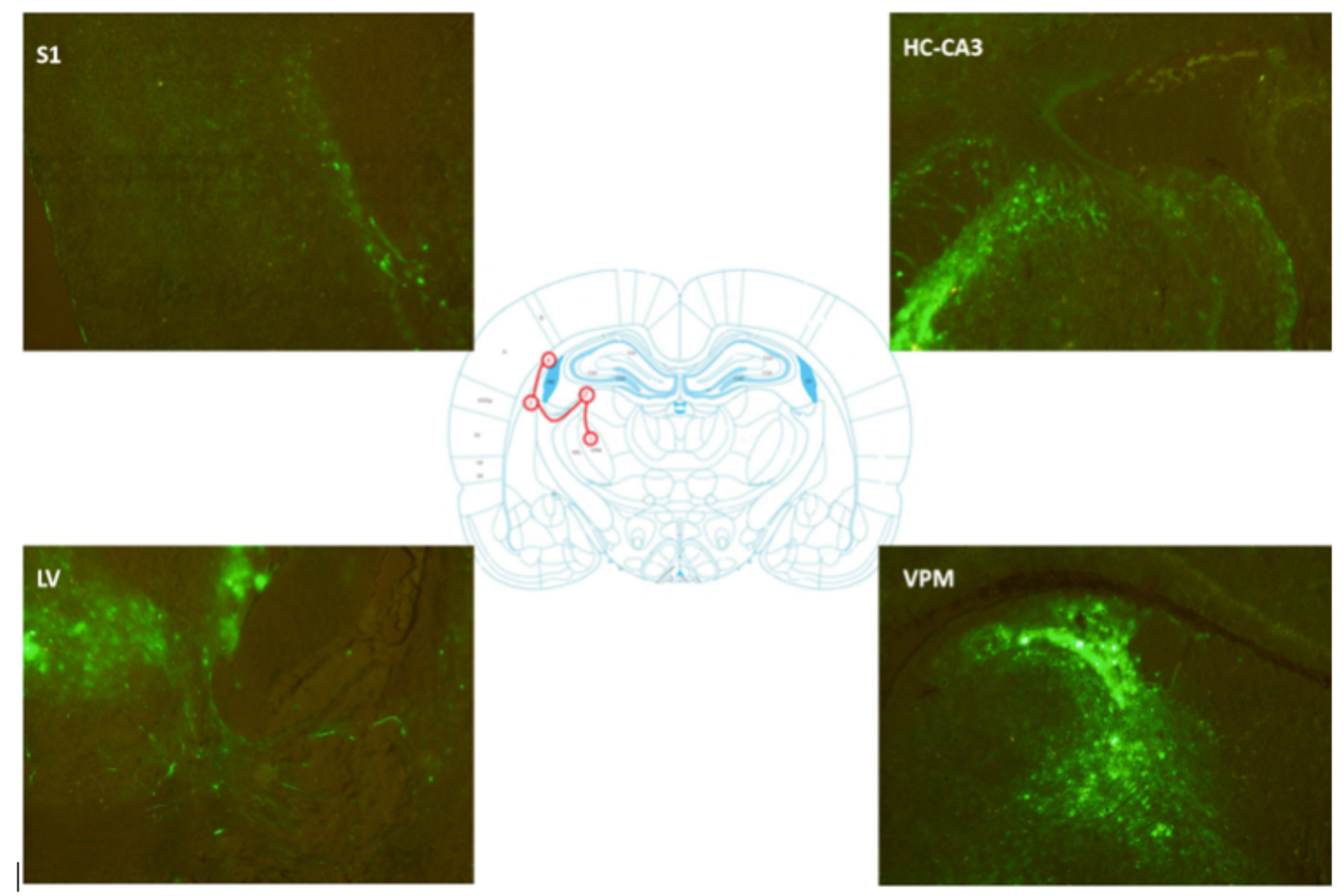

Figure 1

Fluorescence observation site of projected fibers in thalamus and cortex: $₫$ ventromedial nucleus of

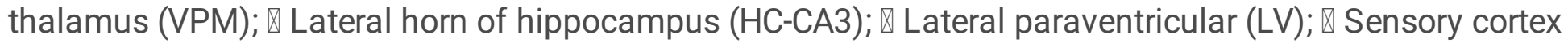
(S1)
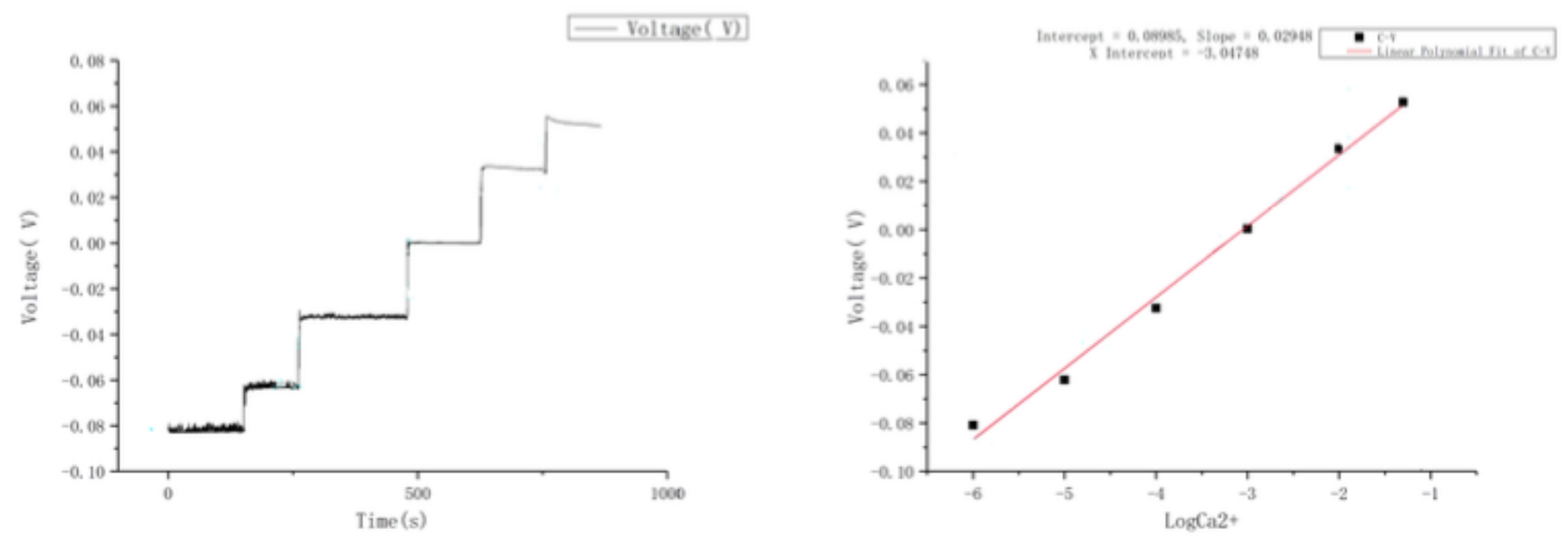

Figure 2 
a. Ca2+ CFMEs electrode was calibrated in a standard concentration of calcium ions solution. The calcium ion electrode was placed in the standard calcium ion concentration solution then quantitative calcium ions were added to test the electrode reactivity to concentration change. b. Voltage-Concentration equation. The concentration of the $\mathrm{Ca} 2+$ solution was defined as the horizontal axis, and the $\mathrm{Ca} 2+$ voltage was defined as the vertical axis. The $\mathrm{Ca} 2+$ concentration is plotted with the corresponding calcium ion voltage (a), with the slope being the Nernst response slope.
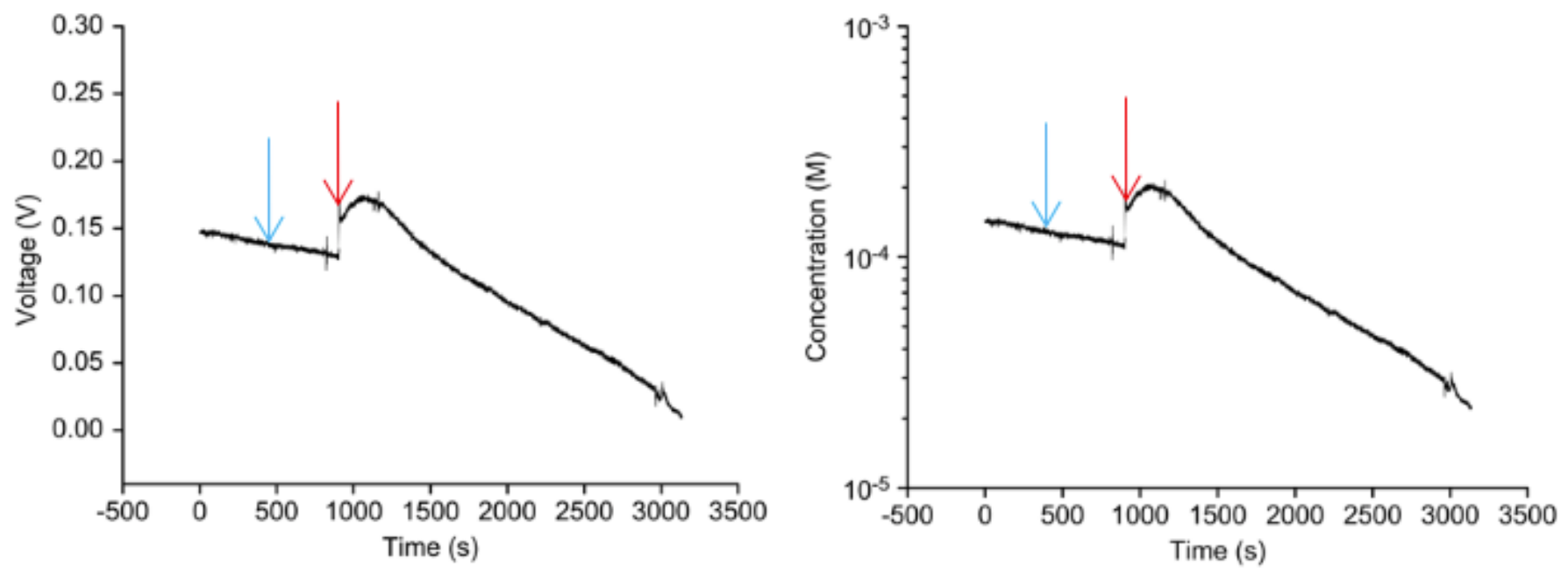

\section{Figure 3}

Response to physiological saline stimulation of the superior sagittal sinus (blue arrow) and to the inflammatory soup stimulation (red arrow) a. Changes in Ca2+ voltage after physiological saline and inflammatory soup stimulation. b. Changes in Ca2+ concentration after physiological saline and inflammatory soup stimulation.
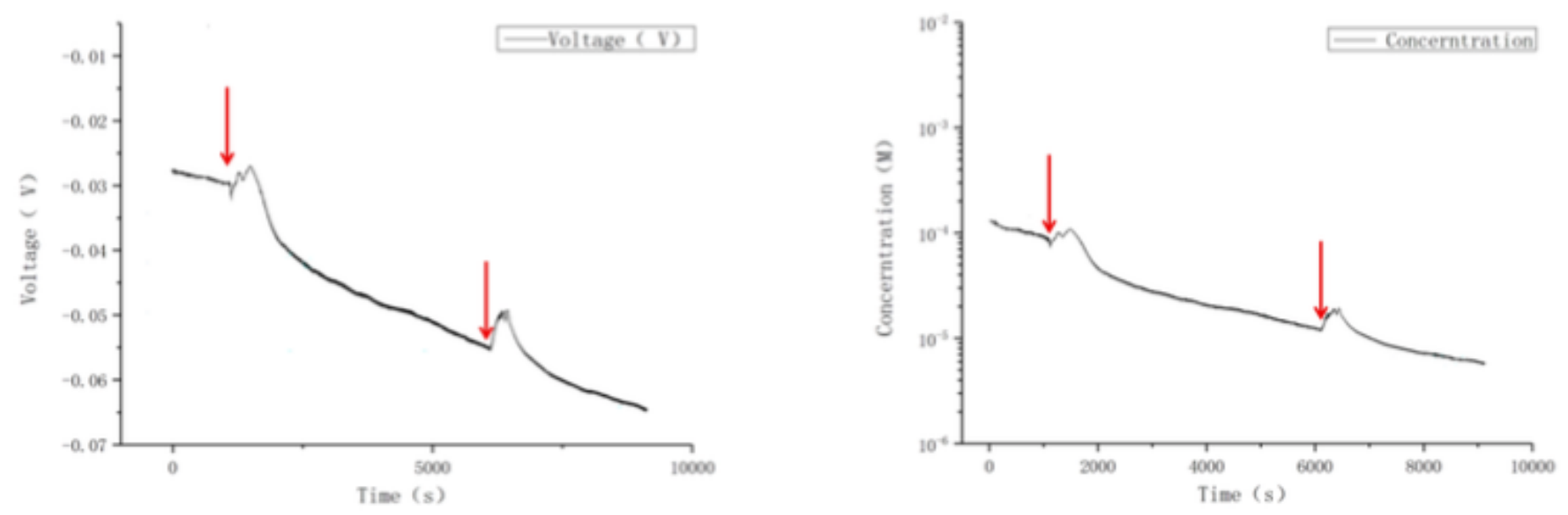

\section{Figure 4}

Response to two inflammatory soup stimulations of the superior sagittal sinus (red arrow) a. Changes in calcium ion voltage after inflammatory soup stimulation. b. Changes in calcium ion concentration after 
inflammatory soup stimulation.
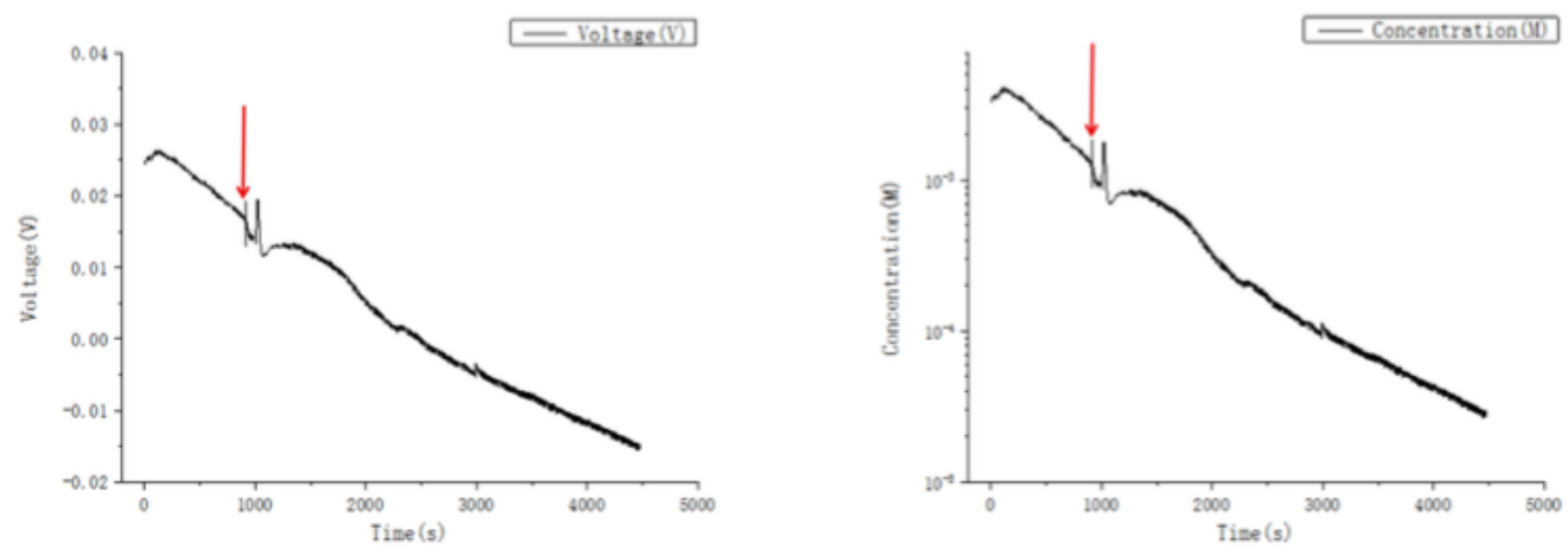

Figure 5

Response to the inflammatory soup stimulation of the superior sagittal sinus of the Nimodipine intervention group (red arrow) a. Changes in Ca2+ voltage after inflammatory soup stimulation. b. Changes in $\mathrm{Ca} 2+$ concentration after inflammatory soup stimulation.
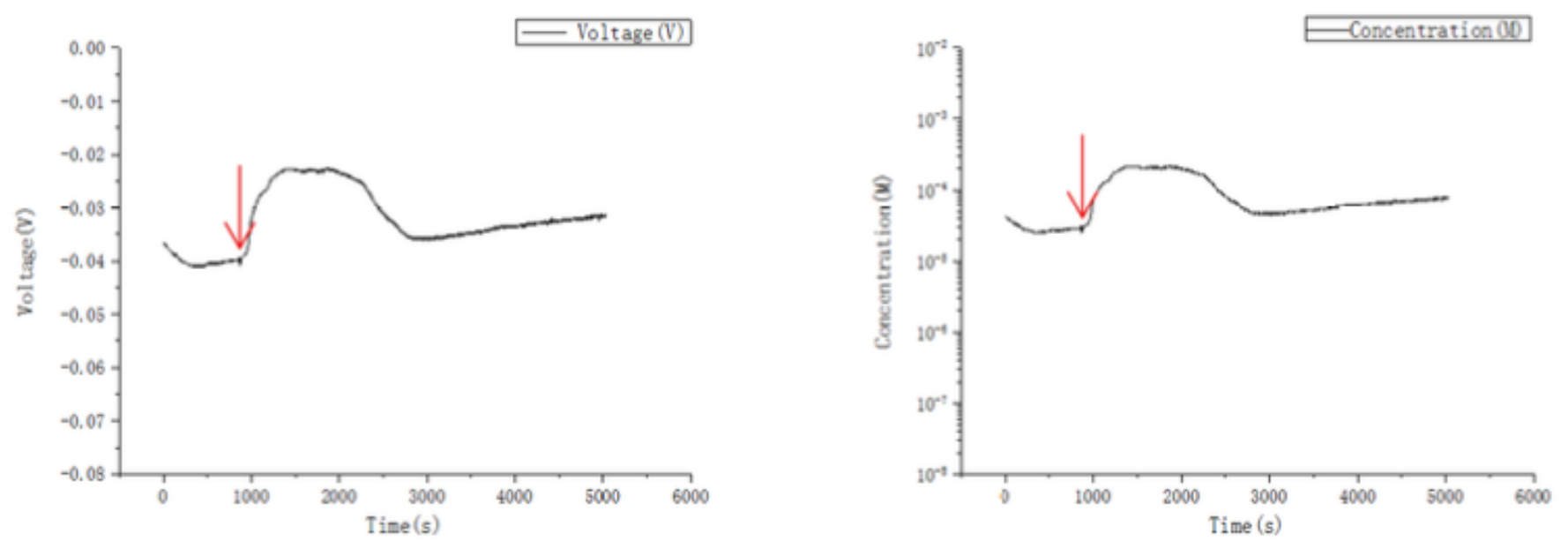

\section{Figure 6}

Response to the inflammatory soup stimulation of the superior sagittal sinus of the Flunarizine intervention group (red arrow) a. Changes in calcium ion voltage after inflammatory soup stimulation. $b$. Changes in calcium ion concentration after inflammatory soup stimulation.

\section{Supplementary Files}

This is a list of supplementary files associated with this preprint. Click to download. 
- ARRIVEGuidelinesChecklist.pdf

Page 19/19 